\title{
Design and Development of Agricultural Drone for Plant Health Analysis
}

\author{
${ }^{1}$ Yogesh Bharambe, ${ }^{2}$ Divesh Singh, ${ }^{3}$ Vedang Binsale \\ ${ }^{1,2,3}$ Student, Dept of Computer Engineering, Thakur College of Engineering and Technology, Mumbai, India
}

\begin{abstract}
Agricultural drones let the farmers observe and analyze the fields/ sectors from the sky. This overview of the sector can reveal several problems like soil variation, irrigation problems, and pest and fungal infections. The mixture of these factors permits the farmer to distinguish between healthy and unhealthy plants, a distinction not always clearly visible. Thus, these views will aid in evaluating crop growth and production. The project is to develop an autonomous flying quadcopter, equipped with a GPS tracking system and programmed to be able to fly autonomously from one location to a different location using GPS coordinates. Its goal is to act as a proof-ofconcept for a small scale autonomous geographical region mapping (agricultural field in this case) and determine plant health using the Visible Atmospherically Resistant Index algorithm.
\end{abstract}

Keywords: Quadcopter, Plant Health, Visible Atmospherically Resistant Index (VARI), Normalized Difference Vegetation Index (NDVI), Unmanned Ariel Vehicles (UAV), Geographical Information System (GIS), GPS Module and Telemetry.

\section{INTRODUCTION}

Current methods in the agricultural sector for monitoring plants' health involve manually visiting each part of the field and analyze the area. However, with the use of Unmanned Aerial Vehicle (UAV) and vegetation index algorithms, farmers can monitor the health of the plants by getting the heat map of the overview of the field from the images captured by the UAV.

Remote sensing supported drones (UAV- Unmanned Aerial Vehicle) are a rapidly developing field of technology, which permits new possibilities for monitoring of the environment. Due to technological advancements, multisensors have been developed which can be carried even by small to medium-sized drones [1]. The UAV applications can comprise precision agriculture, vegetation monitoring and forest monitoring [1] [2] [3]. The Drone-based system enables us to observe the agricultural lands with high resolution, which further enables us to detect even small vegetation anomalies. Such anomalies in vegetation/ plants may be caused by, for example, drought-induced uneven germination and growth, seedling loss caused by flooding, disease outbreak and pest invasion [2].

These systems, coupled with the Vegetation Index algorithms, provide a good estimate of plant health by using various parameters like soil moisture, amount of greenness, etc. [2].

\section{LITERATURE SURVEY}

Nandyal Suvarna - "Agridrone: Automation of Agriculture using IoT": This paper discusses a system that consists of a flying drone fitted with a camera eye to record pictures of crops in a scheduled time. The paper discusses developing an intelligent system to guide agriculturists, by building a database which includes various cases of assorted crops and decisions based on crop image analysis. From captured pictures, the parameters that are planned for analysis by image processing are the extent of greenness in leaf detection, the moisture content in the soil, etc. [1].

Reinecke - "The influence of drone monitoring on crop health and harvest size": This paper explores the advantages of drones and their limitations in agriculture. Various features of drones are analyzed, like how they assist farmers in maximizing their yield by detecting anomalies early and managing the crops by using specific cameras to detect pests and water shortages [2].

J. Primicerio et al - "A flexible unmanned aerial vehicle for precision agriculture": This paper describes a project which involved developing a hexacopter for vineyard management. The system consists of a hexacopter and multispectral camera for capturing vegetation. The vegetation index used is the Normalized Difference Vegetation Index (NDVI). The system has delivered very promising results for its development as a tool for precision agriculture applications in small crop fields [3].

F.G. Costa and et.al - "The use of unmanned aerial vehicles and wireless sensor network in agriculture applications": The paper discusses how UAVs represent technological developments utilized in precision agriculture. They supply high-resolution images of the plants/ crops and 
ISSN (online): 2581-3048

different vegetation indices generate useful outputs for decision-making for farm management. Also, a literature review on the utilization of UAVs in agriculture and specific applications are discussed [4].

E. George, G. Tiwari, R. Yadav, S. Sadana, and E. Peters, "Uav systems for parameter identification in agriculture": This paper discusses parameters that aid in identifying the status of a field from data gathered by the UAV. Some of the parameters discussed are timely irrigation, soil nutrients, etc. The monitoring of these parameters aid in precision agricultural methods. [5].

Ahmad - "Aerial mapping using high-resolution digital camera and unmanned aerial vehicle for Geographical Information System.": This paper focuses on the utilization of a high-resolution camera and a light-weight UAV for acquiring data by capturing digital aerial images. These aerial images were processed using image processing software to supply digital maps and digital ortho photos. The results showed that the accuracy of the sub-meter is often obtained using the employed method. Topographic maps and ortho photos generated were used as an input to the Geographical Information System (GIS) for further image exploration [6].

Gitelson, Anatoly A., et al. "Novel algorithms for remote estimation of vegetation fraction.": This paper discusses various vegetation index algorithms to aid in precision agriculture. The indices discussed are the Visible Atmospherically Resistant Index (VARI), Soil Adjusted Vegetation Index (SAVI), Normalized Difference Vegetation Index (NDVI), Green Atmospherically Resistant Index (GARI), etc. [7].

Santos - "Indoor waypoint UAV navigation using an RGB-D system.": This paper discusses a technique for measuring the approximate orientation and position of UAV, through processing information provided by an RGB-D sensor and an Inertial Measurement Unit (IMU) to examine predefined points in an indoor environment [8].

Roberts - "GPS Guided Autonomous Drone.”: The project is to develop an autonomous flying quadcopter. The drone could fly from location to location using GPS. The drone would help in small scale autonomous aerial delivery like that deployed by Amazon [12].

\section{EASE OF USE}

\section{a) Monitoring Crop Production}

Drones have already proven to be effective at recording the canopy reflectance over vineyards [4]. To measure the growth of crops over a season, aerial images can be taken at the start of the season, at predetermined intervals during the season and just before harvesting to illustrate the growth across the field, as well as highlighting any rows that show signs of stunted growth due to poor irrigation or low initial nitrogen content [4] [5]. Birds may fear a scarecrow for a time, but they will soon realize it is not alive and return in numbers -meaning the only solution is a lethal one. Larger animals like baboons and deer can only be kept away with tall often electrified fences with empty cans to make a noise. Amid in all these preventative measures, farmers may forget about a section of an orchard or field - the consequences of which will only be apparent when it is too late to remedy. Crop diseases can be devastating and classified as bacterial, fungal, or viral. Drones equipped with Infrared cameras can see inside plants, giving a clear image of the condition thereof [3] [5]. If a farmer can detect an infection before it spreads, preventative measures can be taken - like removing the plant -before the infection spreads to neighboring plants. Drones can be equipped with a multispectral camera that can detect the water content underground, which can allow a farmer to determine if a crop row is parched or over hydrated. Farmers can adjust their irrigation until even the water table is created, eliminating water as a source of crop distress. If a multispectral camera is not available, the drone can still take overhead pictures of the fields and highlight areas where plants seem discolored or smaller than the rest of the field. This technique can also be used to detect underground leaks in existing irrigation systems [2] [6].

\section{b) Crime and Damage Reports}

The drone can aid the authorities in identifying the vehicle employed by the criminals, speeding up the investigation. Also, some farmers can utilize drones as guards to monitor entrances and along fences.

\section{c) Forestry}

The Forestry sector can use drone-based region mapping for valuation, monitoring, and research. Hundreds of images taken by drones can be mapped/ stitched together to create wide and high-resolution ortho maps. These ortho maps can then be used for analysis, planning and management via the GIS systems [6].

\section{DRONE SYSTEM ARCHITECTURE}

For this project, we have built a Quadcopter Drone for agriculture surveillance and analysis. The additional functionality/ component is "ublox neo $7 \mathrm{~m}$ GPS with compass" - it is a GPS component with an inbuilt compass in it; it will be used to guide the drone without any manual guidance from any personnel from the organization [2] [7]. 
ISSN (online): 2581-3048

With the help of Telemetry components, the directions to the drone can be given from any android phones through an application. Another component is "Xmate 16 Megapixel Waterproof Camera" - Used for recording the whole agricultural land and take HD Images for further analysis.

After the flight and recording of data of crop, it will be passed through a set of codes. We will use VARI (Vegetation Atmospheric Resistant Index) Algorithm for Analysis of Crop health.

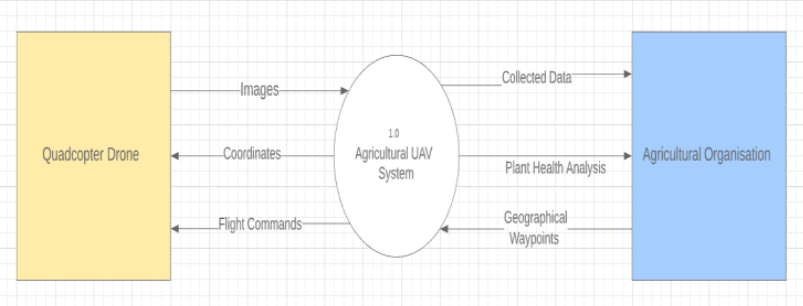

Figure 1: Structural model - DFD Level 0

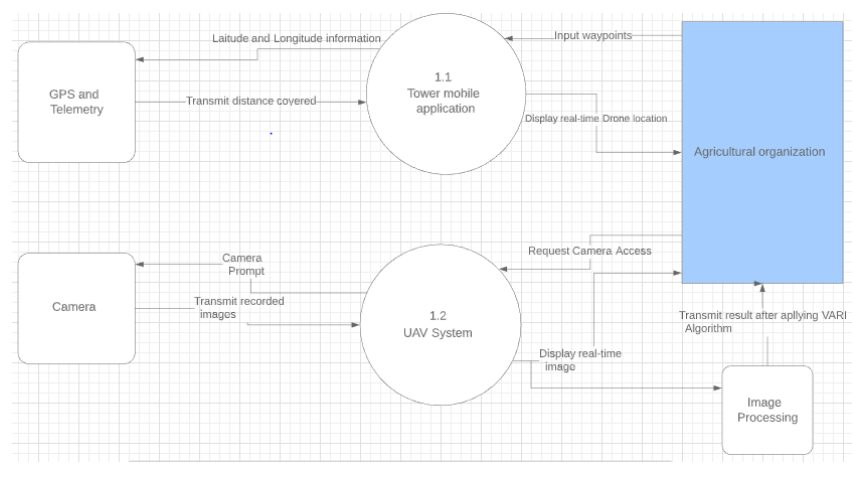

Figure 2: Structural model - DFD Level 1

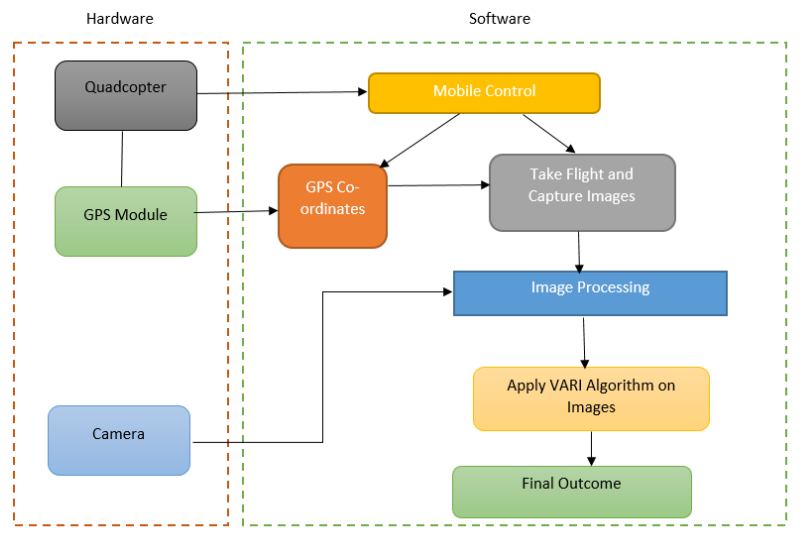

Figure 3: Flow Diagram

\section{a) VARI Algorithm}

VARI algorithm is a vegetation index used to record plants' health. For the VARI algorithm, RGB (standard) cameras, which capture Red, Green, and Blue light, are used for imaging purposes. The Visible Atmospherically Resistant Index (VARI) was designed to work with RGB cameras. VARI is a measure of "how green" a picture is [7]. VARI is only meaningful when working with non-NDVI imagery and is therefore not intended as a replacement for the NDVI algorithm. The Heatmap of RGB images generated by the VARI algorithm allows the identification of crop stress areas in a vegetation field [8].

VARI Index formula can be given as:

$$
V A R I=\frac{\text { Green }- \text { Red }}{\text { Green }+ \text { Red }- \text { Blue }}
$$

We can use drone imagery to assess plant health, as healthy plants reflect light differently than unhealthy plants. Healthier plants reflect greener light than red light, thus they appear green [9].

Plant health algorithms (or vegetation index algorithms) like VARI and NDVI use the data generated by the proportion of sunlight captured across different bands (red, green, blue and sometimes near-infrared) to compute numerical values (between -1 to 1) for every pixel of a given drone image/ map. Maps generated by these algorithms are then assigned a color based on those numerical values, which facilitates early detection of unavoidable plant diseases [10] [11].

\section{b) GPS module and Telemetry}

A GPS module can provide an estimation of your drone's altitude, but the measurements are unreliable and can only offer you a position to within $5 \mathrm{~m}$. However, by combining measurements from other sensors like the barometric sensor and altitude sensor, the flight controller can get a clearer description of what the drone is doing. The key advantage of the GPS module is that it is possible to easily fly your drone autonomously to way-points, enabling the drone to fly on its own from takeoff to landing with the help of the Telemetry module [12].

The telemetry modules are the actual radio devices, which aids in waypoint navigation. These enable the user to feed in the way-points to the drone. The drone then flies on its own following the waypoints.

\section{c) Applications}

- Land surveying

- Cadastral maps

- Topographical surveys

- Land management

- Monitor plant health 
ISSN (online): 2581-3048

Volume 4, Issue 8, pp 6-11, August-2020

- Perform plant count

- Optimize plant ROI

\section{d) Advantages}

- Easy to use

- Cost-effective

- Automated; requires less human interaction due to GPS and Telemetry

- Can be controlled through any Android mobile

- Recorded images pass through VARI algorithm, yielding plant's health

\section{e) Methodology}

For this project, Scrum methodology is used which is an agile software development methodology. By adopting this methodology, every team member can know the progress of the project. Following this model, our method can be described by the following flow graph:

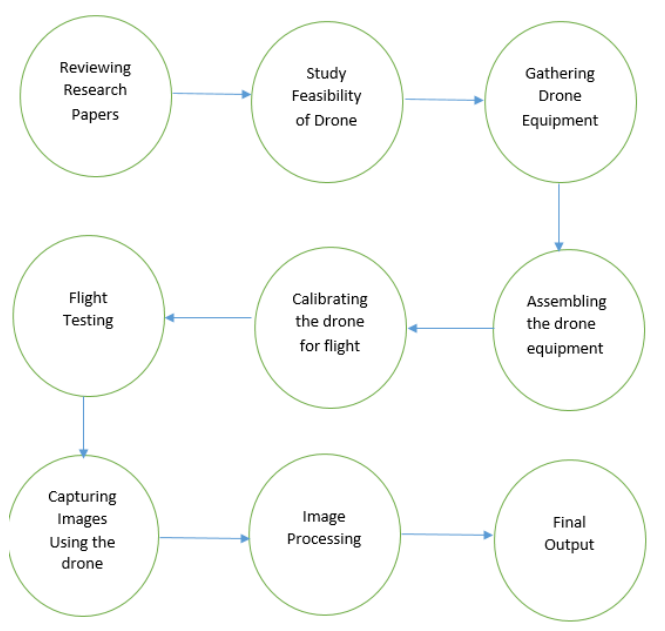

Figure 4: Methodology

\section{f) Drone Connections}

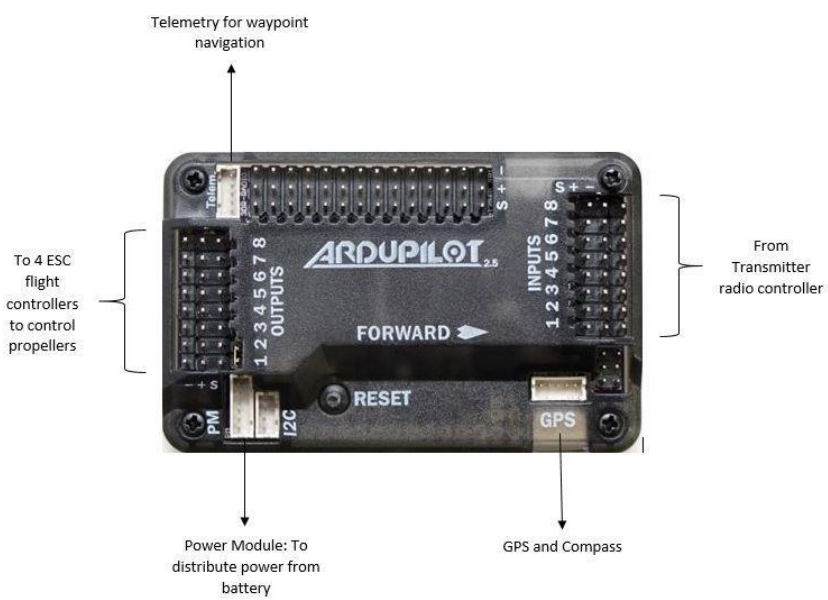

\section{RESULTS AND DISCUSSION}

The key advantage of the quadcopter is the auto-landing capability which reduces the risk factors associated with crashing. It also helps to gather images from a good vantage point to analyze plant quality and health by using the VARI algorithm.

Regarding the functioning of the drone, we have used the Mission Planner application to program the drone to take flight. For the formation of ortho mosaic maps and calculating the VARI indices, we used the QGIS application.

The top view of the final constructed quadcopter is:

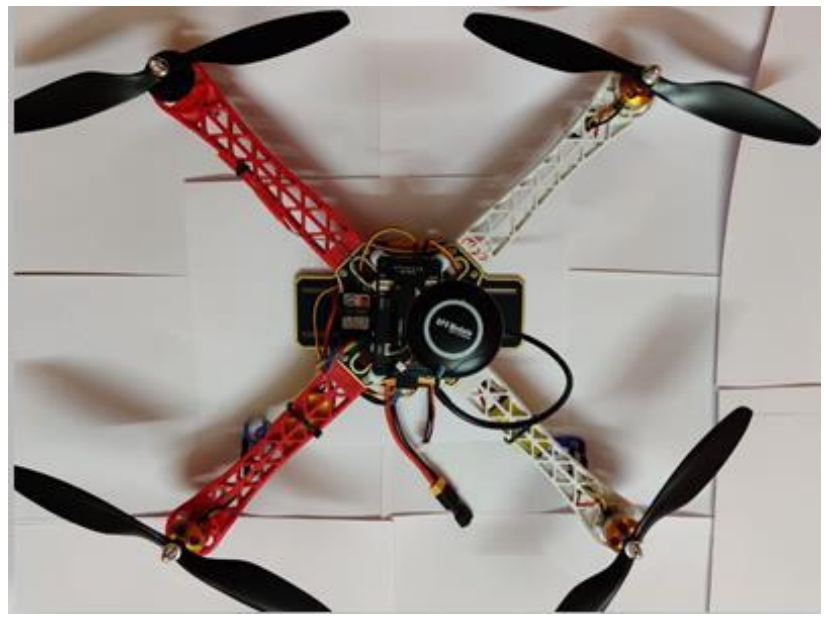

Figure 6: Top View of Quadcopter

To determine plant health, our quadcopter captures images of the fields/ area and processes them via the VARI algorithm. The program outputs a value between -1 to $1 ;-1$ indicating bad plant health and 1 indicating good plant health.

The sample output (health) for good and bad crops, processed by VARI algorithm in the QGIS application are:
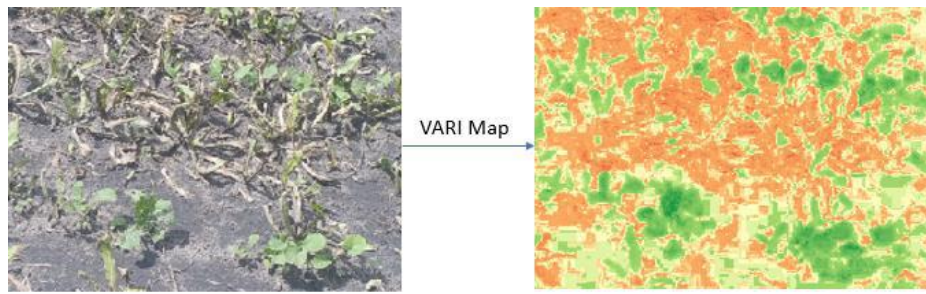

VARI Index: -0.4395178268974735

Figure 7: VARI Index for bad crop field (-0.439) 


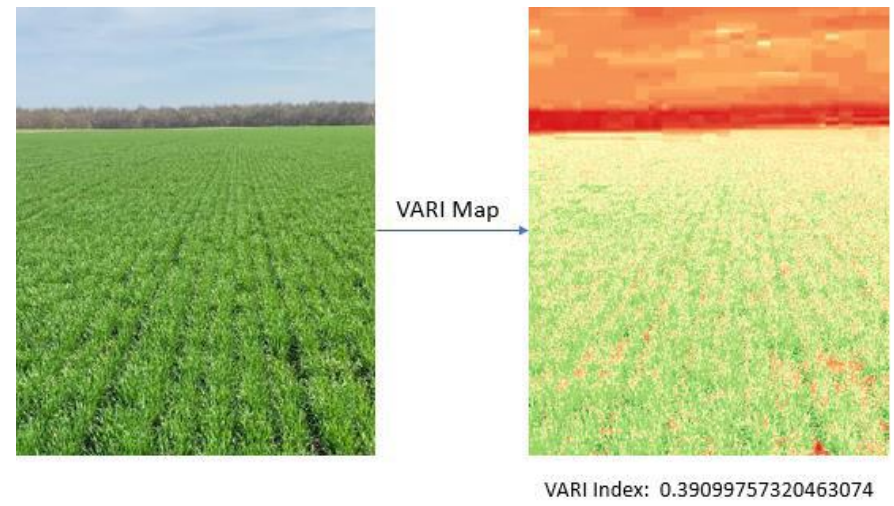

Figure 8: VARI Index for good crop field (0.39)

\section{CONCLUSION}

An autonomous drones such as the one constructed in this project (a Quadcopter) can have many uses in practical applications. Among other things, a GPS guided drone could be used for military and defense purposes.

Hence, there is no doubt that the combination of the UAV and the digital camera could be used and explored further for the GIS application, mapping, detecting deforestation areas and other applications. However, the user must be aware that the area covered by the digital camera is very small. The key advantage of Drone is the auto-landing capability which reduces the risk factors associated with crashing. It also helps to gather images from a good vantage point.

\section{FUTURE SCOPE}

For the future, we will be considering enhancing GPS and Compass for the drone which will increase the range of the drone, which in turn will improve the waypoint navigation.

In the future with the proper budget, we can consider improving the camera quality from RGB to Multispectral Camera. With the use of this camera, we would be able to analyze plant health by using more parameters like soil moisture, this in turn would enable to us use more sophisticated vegetation indices to determine the health with more accuracy. We would also use a more efficient battery for a longer duration of the flight.

\section{ACKNOWLEDGEMENT}

We wish to express sincere thanks to our guide Mrs. Vaishali Nirgude (Assistant Professor, Dept. of Computer Engineering) and our Computer department for providing research opportunities so that we can research about the Agricultural drone and develop the system. We also extend our heartfelt thanks to our colleagues, family members, and well-wishers.

\section{REFERENCES}

[1] Nandyal, Suvarna, Priya S. Khamitkar and Priyanka S. Joshi. "Agridrone: Automation of Agriculture using IoT.", IJISRT Journal (2019).

[2] Reinecke, Marthinus, and Tania Prinsloo. "The influence of drone monitoring on crop health and harvest size." 2017 1st International Conference on Next Generation Computing Applications (NextComp), IEEE (2017).

[3] J. Primicerio et al -, "A flexible unmanned aerial vehicle for precision agriculture." Precision Agriculture 13(4), 517-523, 2012 (Volume: 115, Issue:7).

[4] F.G. Costa and et.al, "The use of unmanned aerial vehicles and wireless sensor network in agriculture applications", IEEE Int. Geoscience and Remote Sensing Symposium, 2012.

[5] E. George, G. Tiwari, R. Yadav, S. Sadana, and E. Peters, "Uav systems for parameter identification in agriculture," in Proc. GHTCSAS, 2013, pp. 270-273.

[6] Ahmad, Anuar, and Abd Manan Samad. "Aerial mapping using high resolution digital camera and unmanned aerial vehicle for Geographical Information System." 2010 6th International Colloquium on Signal Processing \& its Applications, IEEE (2010).

[7] Gitelson, Anatoly A., et al. "Novel algorithms for remote estimation of vegetation fraction." Remote sensing of Environment 80.1 (2002): 76-87.

[8] Santos, Milton CP, Mário Sarcinelli-Filho, and Ricardo Carelli. "Indoor waypoint UAV navigation using a RGB-D system." 2015 Workshop on Research, Education and Development of Unmanned Aerial Systems (RED-UAS), IEEE (2015).

[9] Identifying Crop Variability Patterns. [Online]. Available: https://blog.dronedeploy.com/identifyingcrop-variability-whats-the-difference-between-ndvifalse-ndvi-and-vari-plant-health- 98c380381a33.

[10] QGIS. [Online]. Available: https://github.com/qgis/QGIS

[11] Vegetation Indices. [Online]. Available: http://web.pdx.edu/ nauna/resources/82012_lecture1-vegetationindices.pdf

[12] Roberts and Cameron. "GPS Guided Autonomous Drone", University of Evansville, (2016). 
ISSN (online): 2581-3048

IRJIET

\section{AUTHOR'S BIOGRAPHIES}

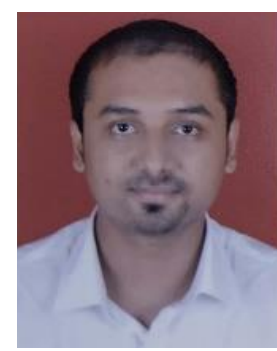

Yogesh Bharambe, Pursuing a Bachelor's degree in Computer Engineering at Thakur College of Engineering and Technology, Mumbai, India

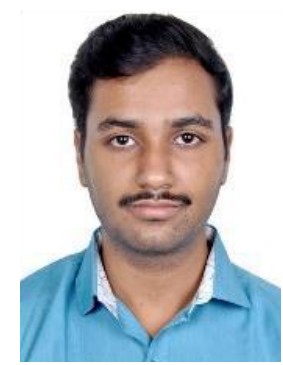

Divesh Singh, Pursuing a Bachelor's degree in Computer Engineering at Thakur College of Engineering and Technology, Mumbai, India

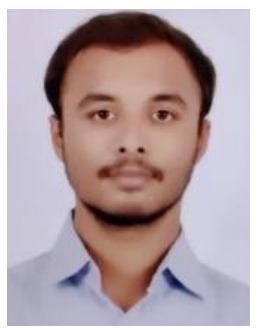

Volume 4, Issue 8, pp 6-11, August-2020 https://doi.org/10.47001/IRJIET/2020.408002

Vedang Binsale, Pursuing a Bachelor's degree in Computer Engineering at Thakur College of Engineering and Technology, Mumbai, India

\section{Citation of this Article:}

Yogesh Bharambe, Divesh Singh, Vedang Binsale, "Design and Development of Agricultural Drone for Plant Health Analysis" Published in International Research Journal of Innovations in Engineering and Technology - IRJIET, Volume 4, Issue 8, pp 6-11, August 2020. https://doi.org/10.47001/IRJIET/2020.408002 